\title{
Synthesis and Biological Evaluation of 2-(3',4',5'-Trimethoxybenzoyl)-3- Amino 5-Aryl Thiophenes as a New Class of Tubulin Inhibitors
}

\author{
Romeo Romagnoli*†, Pier Giovanni Baraldi ${ }^{\dagger}$, Vincent Remusat ${ }^{\dagger}$, Maria Dora Carrion ${ }^{\dagger}$, \\ Carlota Lopez Cara $^{\dagger}$, Delia Preti ${ }^{\dagger}$, Francesca Fruttarolo ${ }^{\dagger}$, Maria Giovanna Pavani ${ }^{\dagger}$, Mojgan \\ Aghazadeh Tabrizi ${ }^{\dagger}$, Manlio Tolome ${ }^{\dagger \dagger}$, Stefania Grimaudo ${ }^{\dagger \dagger}$, Jan Balzarini ${ }^{\Psi}$, Mary Ann \\ Jordan $^{\S}$ and Ernest Hamel ${ }^{\ddagger}$
}

Dipartimento di Scienze Farmaceutiche, Università di Ferrara, 44100 Ferrara, Italy; Divisione di Ematologia e Servizio AIDS, Dipartimento di Oncologia, Policlinico "P. Giaccone”, Università di Palermo, Palermo, Italy; Rega Institute for Medical Research, Laboratory of Virology and Chemotherapy, Minderbroedersstraat 10, B-3000 Leuven, Belgium; Department of Molecular, Cellular and Developmental Biology and Neuroscience Research Institute, University of California Santa Barbara, Santa Barbara, CA, 93106, USA; Toxicology and Pharmacology Branch, Developmental Therapeutics Program, Division of Cancer Treatment and Diagnosis, National Cancer Institute at Frederick, National Institutes of Health, Frederick, Maryland 21702, USA

\section{SUPPORTING INFORMATION}

Characterization of compounds 5a-t, 7a-t and 8, elemental analyses for compounds 5a-t, biological assays. 
Chemistry. Materials and Methods. All actophenones are commercially available and were used as received. ${ }^{1} \mathrm{H}$ NMR spectra were recorded on a Bruker AC 200 spectrometer. Chemical shifts $(\delta)$ are given in ppm upfield from tetramethylsilane as internal standard, and the spectra were recorded in appropriate deuterated solvents, as indicated. Positive-ion electrospray ionization (ESI) mass spectra were recorded on a double-focusing Finnigan MAT 95 instrumental with BE geometry. Melting points ( $\mathrm{mp}$ ) were determined on a BuchiTottoli apparatus and are uncorrected. All products reported showed ${ }^{1} \mathrm{H}$ NMR spectra in agreement with the assigned structures. Elemental analyses were conducted by the Microanalytical Laboratory of the Chemistry Department of the University of Ferrara. All reactions were carried out under an inert atmosphere of dry nitrogen, unless otherwise described. Standard syringe techniques were applied for transferring dry solvents. Reaction courses and product mixtures were routinely monitored by TLC on silica gel (precoated F254 Merck plates) and visualized with aqueous $\mathrm{KMnO}_{4}$. Flash chromatography was performed using 230-400 mesh silica gel and the indicated solvent system. Organic solutions were dried over anhydrous $\mathrm{Na}_{2} \mathrm{SO}_{4}$.

For the characterization $\left({ }^{1} \mathrm{H}-\mathrm{NMR}\right)$ of compounds $\mathbf{7 a}, \mathbf{7 f}, \mathbf{7 i}, \mathbf{7 l}, \mathbf{7 r}$ and $\mathbf{7 t}$ see: Hartmann, H.; Liebscher, J. A simple method for the synthesis of 5-aryl-3-amino-2alkoxycarbonylthiophenes. Synthesis 1984, 3, 275-276.

For the characterization $\left({ }^{1} \mathrm{H}-\mathrm{NMR}\right)$ of compounds $\mathbf{7 c}, \mathbf{7 e}, \mathbf{7 m}, \mathbf{7 n}$ and $\mathbf{7 s}$ see: Andersen, H. S.; Olsen, O. H.; Iversen, L. F.; Sorensen, A. L. P.; Mortensen, S. B.; Christensen, M. S.; Branner, S.; Hansen, T. K.; Lau, J. F.; Jeppesen, L.; Moran, E. J.; Su, J.; Bakir, F.; Judge, L.; Shahbaz, M.; Collins, T.; Vo, T.; Newman, M. J.; Ripka, W. C.; Moller, Niels P. H. Discovery and SAR of a novel selective and orally bioavailable nonpeptide classical 
competitive inhibitor class of protein-tyrosine phosphatase 1B. J. Med. Chem. 2002, 45, 4443-4459.

For the characterization $\left({ }^{1} \mathrm{H}-\mathrm{NMR}\right)$ of compound 7k see: Hartmann, H.; Liebscher, J. A facile synthesis of 5-aminopyrazoles by reaction of b-chloro cinnamonitriles with hydrazine hydrate. Synthesis 1984, 3, 276-277.

3-Chloro-3-thiophen-2-yl-acrylonitrile (7b). Following general procedure A, compound 7b was purified by chromatography eluting with petroleum ether-ethyl acetate (9:1). Yellow oil, yield: $30 \% .{ }^{1} \mathrm{H}$ NMR $\left(\mathrm{CDCl}_{3}\right) \delta: 5.91(\mathrm{~s}, 1 \mathrm{H}), 7.11(\mathrm{dd}, J=5.4$ and $4.0 \mathrm{~Hz}, 1 \mathrm{H}), 7.52(\mathrm{~d}$, $J=5.4 \mathrm{~Hz}, 1 \mathrm{H}), 7.70(\mathrm{~m}, 1 \mathrm{H})$.

3-Chloro-3-pyridin-4-yl-acrylonitrile (7d). Following general procedure A, compound 7d was purified by chromatography eluting with ethyl acetate-dichloromethane (6:4). Grey powder, yield: $65 \%, \mathrm{mp} 114-116{ }^{\circ} \mathrm{C} .{ }^{1} \mathrm{H}$ NMR $\left(\mathrm{CDCl}_{3}\right) \delta: 6.22(\mathrm{~s}, 1 \mathrm{H}), 7.52(\mathrm{~d}, J=4.4 \mathrm{~Hz}$, 2H), $8.77(\mathrm{~d}, J=4.4 \mathrm{~Hz}, 2 \mathrm{H})$.

3-Chloro-3-(2-chlorophenyl)-acrylonitrile (7g). Following general procedure $\mathbf{A}$, compound $7 \mathbf{g}$ was purified by column chromatography eluting with petroleum ether-ethyl acetate $(9: 1)$. Yellow oil, yield: $65 \% .{ }^{1} \mathrm{H}$ NMR $\left(\mathrm{CDCl}_{3}\right) \delta: 5.91(\mathrm{~s}, 1 \mathrm{H}), 7.43(\mathrm{~m}, 4 \mathrm{H})$.

3-Chloro-3-(3,4-dichlorophenyl)-acrylonitrile (7h). Following general procedure A, compound $\mathbf{7 h}$ was purified by column chromatography eluting with petroleum ether-ethyl acetate (9.5:0.5). Pale yellow powder, yield: $78 \%, \mathrm{mp} 107-109{ }^{\circ} \mathrm{C} .{ }^{1} \mathrm{H}$ NMR $\left(\mathrm{CDCl}_{3}\right) \delta: 6.03$ (s, 1H), $7.50(\mathrm{~d}, J=8.6 \mathrm{~Hz}, 1 \mathrm{H}), 7.56(\mathrm{~d}, J=8.6 \mathrm{~Hz}, 1 \mathrm{H}), 7.76(\mathrm{~m}, 1 \mathrm{H})$.

3-Chloro-3-(4-iodophenyl)-acrylonitrile (7j). Following general procedure A, compound 7j was purified by column chromatography eluting with petroleum ether-ethyl ether $(9: 1)$. 
Yellow powder, yield: $71 \%$. mp 112-114 ${ }^{\circ} \mathrm{C} .{ }^{1} \mathrm{H}$ NMR $\left(\mathrm{CDCl}_{3}\right)$ \&: $5.96(\mathrm{~s}, 1 \mathrm{H}), 7.29$ (d, $J=8.6 \mathrm{~Hz}, 2 \mathrm{H}), 7.74(\mathrm{~d}, J=8.6 \mathrm{~Hz}, 2 \mathrm{H})$.

3-Chloro-3-(3,4,5-trimethoxyphenyl)-acrylonitrile (7o). Following general procedure A, compound 7o was purified by column chromatography eluting with petroleum ether-ethyl acetate (9:1). Pale brown powder, yield: $49 \%, \mathrm{mp} 125-127{ }^{\circ} \mathrm{C} .{ }^{1} \mathrm{H}$ NMR $\left(\mathrm{CDCl}_{3}\right)$ $\delta: 3.90$ (s, $9 \mathrm{H}), 5.95(\mathrm{~s}, 1 \mathrm{H}), 6.85(\mathrm{~s}, 2 \mathrm{H})$.

3-Chloro-3-(4-ethoxyphenyl)-acrylonitrile (7p). Following general procedure A, compound 7p was purified by column chromatography eluting with petroleum ether-ethyl acetate $(9: 1)$. Yellow oil, yield: 55\%. ${ }^{1} \mathrm{H}$ NMR $\left(\mathrm{CDCl}_{3}\right) \delta: 1.43(\mathrm{t}, J=7.2 \mathrm{~Hz}, 3 \mathrm{H}), 4.06(\mathrm{q}, J=7.2 \mathrm{~Hz}, 2 \mathrm{H})$, $5.89(\mathrm{~s}, 1 \mathrm{H}), 6.89$ (d, J=6.8 Hz, 2H), $7.60(\mathrm{~d}, J=6.8 \mathrm{~Hz}, 2 \mathrm{H})$.

3-Chloro-3-(4-trifluoromethylphenyl)-acrylonitrile (7q). Following general procedure A, compound $\mathbf{7 q}$ was purified by chromatography eluting with petroleum ether-ethyl acetate (8.5:1.5). Yellow oil, yield: $73 \% .{ }^{1} \mathrm{H}$ NMR $\left(\mathrm{CDCl}_{3}\right) \delta: 6.10(\mathrm{~s}, 1 \mathrm{H}), 7.68(\mathrm{~d}, J=6.2 \mathrm{~Hz}, 2 \mathrm{H})$, $7.80(\mathrm{~d}, J=6.2 \mathrm{~Hz}, 2 \mathrm{H})$.

[3-Amino-5-phenyl-thiophen-2-yl]-(3,4,5-trimethoxyphenyl)-methanone (5a). Following procedure B, compound 5a was isolated as a yellow oil. Yield: $57 \%$. ${ }^{1} \mathrm{H}$ NMR $\left(\mathrm{CDCl}_{3}\right) \delta$ : $3.92(\mathrm{~s}, 3 \mathrm{H}), 3.93(\mathrm{~s}, 6 \mathrm{H}), 5.10(\mathrm{bs}, 2 \mathrm{H}), 6.86(\mathrm{~s}, 1 \mathrm{H}), 7.14(\mathrm{~s}, 2 \mathrm{H}), 7.39(\mathrm{~m}, 3 \mathrm{H}), 7.60$ (m, 2H). MS-ESI $(m / z): 370.4[\mathrm{M}+1]^{+}$. Anal. $\left(\mathrm{C}_{20} \mathrm{H}_{19} \mathrm{NO}_{4} \mathrm{~S}\right): \mathrm{C}, \mathrm{H}, \mathrm{N}$.

(4-Amino-[2,2']-bithiophenyl-5-yl)-(3,4,5-trimethoxyphenyl)-methanone (5b). Following general procedure $\mathrm{B}$, compound $\mathbf{5 b}$ was purified by flash chromatography eluting with dichloromethane-ethyl acetate (9:1). Yellow solid, yield: 64\%; mp 115-117 ${ }^{\circ} \mathrm{C}$. ${ }^{1} \mathrm{H}$ NMR 


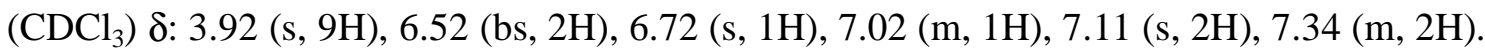
$\operatorname{MS}-\operatorname{ESI}(m / z): 376.2[\mathrm{M}+1]^{+}$. Anal. $\left(\mathrm{C}_{18} \mathrm{H}_{17} \mathrm{NO}_{4} \mathrm{~S}_{2}\right): \mathrm{C}, \mathrm{H}, \mathrm{N}$.

(4-Amino-[2,3']-bithiophenyl-5-yl)-(3,4,5-trimethoxyphenyl)-methanone (5c). Following general procedure B, compound $\mathbf{5 c}$ was isolated as a pale yellow solid. Yield: 66\%; mp 134-

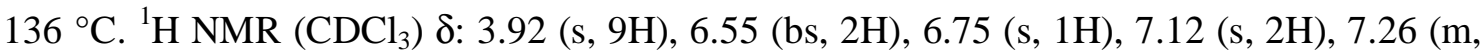
1H), $7.40(\mathrm{~m}, 1 \mathrm{H}), 7.55(\mathrm{~m}, 1 \mathrm{H})$. MS-ESI $(\mathrm{m} / \mathrm{z}): 376.2[\mathrm{M}+1]^{+}$. Anal. $\left(\mathrm{C}_{18} \mathrm{H}_{17} \mathrm{NO}_{4} \mathrm{~S}_{2}\right): \mathrm{C}, \mathrm{H}$, N.

\section{(3-Amino-5-pyridin-4-yl-thiophen-2-yl)-(3,4,5-trimethoxyphenyl)-methanone}

(5d).

Following general procedure B, compound 5d was purified by column chromatography eluting with ethyl acetate. Orange solid, yield: 50\%; mp 187-189 ${ }^{\circ} \mathrm{C} .{ }^{1} \mathrm{H}$ NMR $\left(\mathrm{CDCl}_{3}\right) \delta$ : $3.92(\mathrm{~s}, 9 \mathrm{H}), 6.50(\mathrm{bs}, 2 \mathrm{H}), 7.01(\mathrm{~s}, 1 \mathrm{H}), 7.12(\mathrm{~s}, 2 \mathrm{H}), 7.44(\mathrm{~d}, \mathrm{~J}=6 \mathrm{~Hz}, 2 \mathrm{H}), 8.63$ (d, J=6 Hz, 2H). MS-ESI $(m / z): 371.3[\mathrm{M}+1]^{+}$. Anal. $\left(\mathrm{C}_{19} \mathrm{H}_{18} \mathrm{~N}_{2} \mathrm{O}_{4} \mathrm{~S}\right): \mathrm{C}, \mathrm{H}, \mathrm{N}$.

[3-Amino-5-(4-fluorophenyl)-thiophen-2-yl]-(3,4,5-trimethoxyphenyl)-methanone (5e). Following general procedure B, compound 5e was isolated as a yellow solid. Yield: 49\%, mp 191-193 ${ }^{\circ} \mathrm{C} .{ }^{1} \mathrm{H}$ NMR $\left(\mathrm{CDCl}_{3}\right)$ $\delta: 3.92$ (s, 9H), 6.52 (bs, 2H), 6.78 (s, 1H), 7.09 (t, J=8.4 Hz, 2H), $7.13(\mathrm{~s}, 2 \mathrm{H}), 7.57(\mathrm{~m}, 2 \mathrm{H})$. MS-ESI $(\mathrm{m} / \mathrm{z}): 388.2[\mathrm{M}+1]^{+}$. Anal. $\left(\mathrm{C}_{20} \mathrm{H}_{18} \mathrm{FNO}_{4} \mathrm{~S}\right): \mathrm{C}, \mathrm{H}$, N.

[3-Amino-5-(4-chlorophenyl)-thiophen-2-yl]-(3,4,5-trimethoxyphenyl)-methanone (5f). Following general procedure B, compound $\mathbf{5 f}$ was isolated as a yellow solid. Yield: $58 \%$, mp

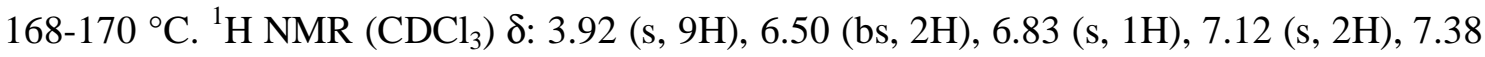
$(\mathrm{d}, \mathrm{J}=6.4 \mathrm{~Hz}, 2 \mathrm{H}), 7.53(\mathrm{~d}, \mathrm{~J}=6.4 \mathrm{~Hz}, 2 \mathrm{H})$. MS-ESI $(\mathrm{m} / \mathrm{z}): 404.3[\mathrm{M}+1]^{+}$. Anal. $\left(\mathrm{C}_{20} \mathrm{H}_{18} \mathrm{ClNO}_{4} \mathrm{~S}\right): \mathrm{C}, \mathrm{H}, \mathrm{N}$. 
[3-Amino-5-(2-chlorophenyl)-thiophen-2-yl]-(3,4,5-trimethoxyphenyl)-methanone (5g).

Following general procedure B, compound $\mathbf{5 g}$ was purified by column chromatography eluting with dichloromethane-ethyl acetate (9:1). Yellow solid, yield: $25 \%$; mp $46-48{ }^{\circ} \mathrm{C} .{ }^{1} \mathrm{H}$ NMR $\left(\mathrm{CDCl}_{3}\right) \delta: 3.90$ (s, 9H), $6.52(\mathrm{bs}, 2 \mathrm{H}), 6.91(\mathrm{~s}, 1 \mathrm{H}), 7.14(\mathrm{~s}, 2 \mathrm{H}), 7.24(\mathrm{~m}, 2 \mathrm{H}), 7.42$ (m, 2H). MS-ESI $(m / z): 404.2[\mathrm{M}+1]^{+}$. Anal. $\left(\mathrm{C}_{20} \mathrm{H}_{18} \mathrm{ClNO}_{4} \mathrm{~S}\right): \mathrm{C}, \mathrm{H}, \mathrm{N}$.

\section{[3-Amino-5-(3,4-dichlorophenyl)-thiophen-2-yl]-(3,4,5-trimethoxyphenyl)-methanone}

(5h). Following general procedure B, compound $\mathbf{5 h}$ was isolated as a yellow solid. Yield: $68 \%$; mp 157-159 ${ }^{\circ} \mathrm{C} .{ }^{1} \mathrm{H}$ NMR $\left(\mathrm{CDCl}_{3}\right) \delta: 3.92(\mathrm{~s}, 9 \mathrm{H}), 6.55(\mathrm{bs}, 2 \mathrm{H}), 6.84(\mathrm{~s}, 1 \mathrm{H}), 7.11$ (s, 2H), $7.40(\mathrm{~m}, 2 \mathrm{H}), 7.68(\mathrm{~m}, 1 \mathrm{H})$. MS-ESI $(\mathrm{m} / \mathrm{z}): 438.2[\mathrm{M}+1]^{+}$. Anal. $\left(\mathrm{C}_{20} \mathrm{H}_{17} \mathrm{Cl}_{2} \mathrm{NO}_{4} \mathrm{~S}\right): \mathrm{C}$, $\mathrm{H}, \mathrm{N}$.

[3-Amino-5-(4-bromophenyl)-thiophen-2-yl]-(3,4,5-trimethoxyphenyl)-methanone (5i). Following general procedure B, compound $\mathbf{5 i}$ was isolated as a yellow solid. Yield: $27 \%$, mp 125-127 ${ }^{\circ} \mathrm{C} .{ }^{1} \mathrm{H}$ NMR $\left(\mathrm{CDCl}_{3}\right)$ \&: 3.93 (s, 9H), 6.55 (bs, 2H), 6.84 (s, 1H), 7.13 (s, 2H), 7.44 $(\mathrm{d}, \mathrm{J}=8.4 \mathrm{~Hz}, 2 \mathrm{H}), 7.57(\mathrm{~d}, \mathrm{~J}=8.4 \mathrm{~Hz}, 2 \mathrm{H})$. MS-ESI $(\mathrm{m} / \mathrm{z}): 451.3[\mathrm{M}+1]^{+}$. Anal. $\left(\mathrm{C}_{20} \mathrm{H}_{18} \mathrm{BrNO}_{4} \mathrm{~S}\right): \mathrm{C}, \mathrm{H}, \mathrm{N}$.

Following general procedure B, compound $\mathbf{5 j}$ was isolated as a yellow solid. Yield: $34 \%$; mp 201-203 ${ }^{\circ} \mathrm{C} .{ }^{1} \mathrm{H}$ NMR $\left(\mathrm{CDCl}_{3}\right)$ $\delta: 3.91$ (s, 9H), 6.57 (bs, 2H), 6.84 (s, 1H), 7.11 (s, 2H), 7.30 $(\mathrm{d}, \mathrm{J}=8.4 \mathrm{~Hz}, 2 \mathrm{H}), 7.72(\mathrm{~d}, \mathrm{~J}=8.4 \mathrm{~Hz}, 2 \mathrm{H})$. MS-ESI $(\mathrm{m} / \mathrm{z}): 496.3[\mathrm{M}+1]^{+}$. Anal. $\left(\mathrm{C}_{20} \mathrm{H}_{18} \mathrm{INO}_{4} \mathrm{~S}\right): \mathrm{C}, \mathrm{H}, \mathrm{N}$.

[3-Amino-5-(4-methylphenyl)-thiophen-2-yl]-(3,4,5-trimethoxyphenyl)-methanone (5k). Following general procedure B, compound 5k was isolated as a yellow solid. Yield: $48 \%$; mp 


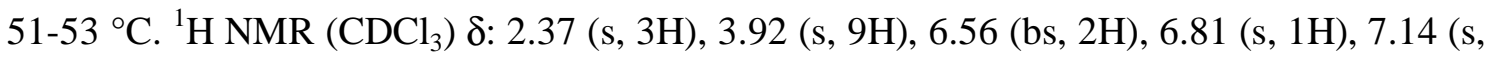
2H), $7.20(\mathrm{~d}, \mathrm{~J}=8.4 \mathrm{~Hz}, 2 \mathrm{H}), 7.50(\mathrm{~d}, \mathrm{~J}=8.4 \mathrm{~Hz}, 2 \mathrm{H})$. MS-ESI $(\mathrm{m} / \mathrm{z}): 384.1[\mathrm{M}+1]^{+}$. Anal. $\left(\mathrm{C}_{21} \mathrm{H}_{21} \mathrm{NO}_{4} \mathrm{~S}\right): \mathrm{C}, \mathrm{H}, \mathrm{N}$.

\section{[3-Amino-5-(4-methoxyphenyl)-thiophen-2-yl]-(3,4,5-trimethoxyphenyl)-methanone}

(51). Following general procedure B, compound $\mathbf{5 l}$ was isolated as a pale yellow solid. Yield: 61\%; mp 54-56 ${ }^{\circ} \mathrm{C} .{ }^{1} \mathrm{H}$ NMR $\left(\mathrm{CDCl}_{3}\right) \delta: 3.83$ (s, 3H), $3.92(\mathrm{~s}, 9 \mathrm{H}), 6.53$ (bs, 2H), 6.74 (s, 1H), $6.91(\mathrm{~d}, \mathrm{~J}=6.8 \mathrm{~Hz}, 2 \mathrm{H}), 7.14(\mathrm{~s}, 2 \mathrm{H}), 7.53(\mathrm{~d}, \mathrm{~J}=6.8 \mathrm{~Hz}, 2 \mathrm{H}) . \operatorname{MS}-\mathrm{ESI}(\mathrm{m} / \mathrm{z}): 400.4[\mathrm{M}+$ $1]^{+}$. Anal. $\left(\mathrm{C}_{21} \mathrm{H}_{21} \mathrm{NO}_{5} \mathrm{~S}\right): \mathrm{C}, \mathrm{H}, \mathrm{N}$.

\section{[3-Amino-5-(3-methoxyphenyl)-thiophen-2-yl]-(3,4,5-trimethoxyphenyl)-methanone}

(5m). Following general procedure B, compound $\mathbf{5 m}$ was purified by column chromatography eluting with dichloromethane-ethyl acetate (9:1). Yellow solid, yield: 29\%; mp: $41-43{ }^{\circ} \mathrm{C} .{ }^{1} \mathrm{H}$ NMR $\left(\mathrm{CDCl}_{3}\right)$ $\delta: 3.84$ (s, 3H), 3.91 (s, 3H), 3.92 (s, 6H), 6.51 (bs, 2H), $6.84(\mathrm{~s}, 1 \mathrm{H}), 6.92(\mathrm{~m}, 1 \mathrm{H}), 7.14(\mathrm{~s}, 2 \mathrm{H}), 7.28(\mathrm{~m}, 3 \mathrm{H}) . \mathrm{MS}-\mathrm{ESI}(\mathrm{m} / \mathrm{z}): 400.3[\mathrm{M}+1]^{+}$. Anal. $\left(\mathrm{C}_{21} \mathrm{H}_{21} \mathrm{NO}_{5} \mathrm{~S}\right): \mathrm{C}, \mathrm{H}, \mathrm{N}$.

\section{[3-Amino-5-(3,4-dimethoxyphenyl)-thiophen-2-yl]-(3,4,5-trimethoxyphenyl)-methanone}

(5n). Following general procedure B, compound 5n was purified by column chromatography eluting with dichloromethane-ethyl acetate (9:1). Yellow solid, yield: $16 \%$; mp: $62-64{ }^{\circ} \mathrm{C} .{ }^{1} \mathrm{H}$

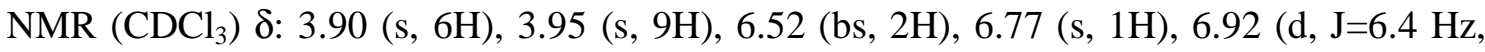
1H), $7.03(\mathrm{~s}, 1 \mathrm{H}), 7.12(\mathrm{~s}, 2 \mathrm{H}), 7.23(\mathrm{~d}, \mathrm{~J}=6.4 \mathrm{~Hz}, 1 \mathrm{H})$. MS-ESI $(\mathrm{m} / \mathrm{z}): 430.3[\mathrm{M}+1]^{+}$. Anal. $\left(\mathrm{C}_{22} \mathrm{H}_{23} \mathrm{NO}_{6} \mathrm{~S}\right): \mathrm{C}, \mathrm{H}, \mathrm{N}$.

\section{[3-Amino-5-(3,4,5-trimethoxyphenyl)-thiophen-2-yl]-(3,4,5-trimethoxyphenyl)-}

methanone (5o). Following general procedure B, compound 5o was isolated as a yellow 


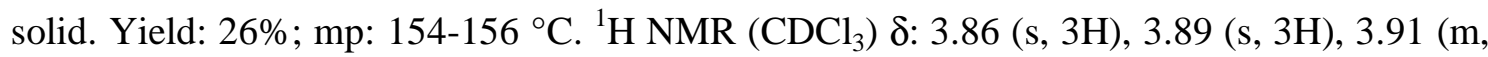
12H), $6.52(\mathrm{bs}, 2 \mathrm{H}), 6.78(\mathrm{~s}, 2 \mathrm{H}), 6.79(\mathrm{~s}, 2 \mathrm{H}), 7.11(\mathrm{~s}, 1 \mathrm{H})$. MS-ESI $(\mathrm{m} / \mathrm{z}): 460.6[\mathrm{M}+1]^{+}$. Anal. $\left(\mathrm{C}_{23} \mathrm{H}_{25} \mathrm{NO}_{7} \mathrm{~S}\right): \mathrm{C}, \mathrm{H}, \mathrm{N}$.

[3-Amino-5-(4-ethoxyphenyl)-thiophen-2-yl]-(3,4,5-trimethoxyphenyl)-methanone (5p). Following general procedure B, compound 5p was purified by column chromatography eluting with dichloromethane-ethyl acetate (9:1). Yellow solid, yield: $28 \%$; mp 137-139 ${ }^{\circ} \mathrm{C}$. ${ }^{1} \mathrm{H}$ NMR $\left(\mathrm{CDCl}_{3}\right) \delta: 1.43(\mathrm{t}, J=7.0 \mathrm{~Hz}, 3 \mathrm{H}), 3.92(\mathrm{~s}, 9 \mathrm{H}), 4.06(\mathrm{q}, J=7.0 \mathrm{~Hz}, 2 \mathrm{H}), 6.51(\mathrm{bs}$, 2H), $6.76(\mathrm{~s}, 1 \mathrm{H}), 6.87$ (d, J=8.8 Hz, 2H), $7.13(\mathrm{~s}, 2 \mathrm{H}), 7.55$ (d, J=8.8 Hz, 2H). MS-ESI $(m / z): 414.3[\mathrm{M}+1]^{+}$. Anal. $\left(\mathrm{C}_{22} \mathrm{H}_{23} \mathrm{NO}_{5} \mathrm{~S}\right): \mathrm{C}, \mathrm{H}, \mathrm{N}$.

\section{[3-Amino-5-(4-trifluoromethylphenyl)-thiophen-2-yl]-(3,4,5-trimethoxyphenyl)-}

methanone (5q). Following general procedure B, compound $\mathbf{5 q}$ was isolated as an orange solid. Yield: 59\%; mp 156-158 ${ }^{\circ} \mathrm{C} .{ }^{1} \mathrm{H}$ NMR $\left(\mathrm{CDCl}_{3}\right)$ $\delta: 3.92$ (s, 9H), 6.56 (bs, 2H), 6.92 (s, 1H), $7.13(\mathrm{~s}, 2 \mathrm{H}) .7 .68(\mathrm{~m}, 4 \mathrm{H}) . \operatorname{MS}-\mathrm{ESI}(\mathrm{m} / \mathrm{z}): 438.2[\mathrm{M}+1]^{+}$. Anal. $\left(\mathrm{C}_{21} \mathrm{H}_{18} \mathrm{~F}_{3} \mathrm{NO}_{4} \mathrm{~S}\right): \mathrm{C}, \mathrm{H}$, N.

\section{[3-Amino-5-(4-nitrophenyl)-thiophen-2-yl]-(3,4,5-trimethoxyphenyl)-methanone $\quad(5 r)$.}

Following general procedure B, compound $\mathbf{5 r}$ was isolated as a red-brown solid. Yield: 40\%, mp 220-222 ${ }^{\circ} \mathrm{C} .{ }^{1} \mathrm{H}$ NMR $\left(\mathrm{CDCl}_{3}\right)$ \&: 3.93 (s, 9H), 6.54 (bs, 2H), 6.98 (s, 1H), 7.12 (s, 2H), $7.76(\mathrm{~d}, \mathrm{~J}=8.8 \mathrm{~Hz}, 2 \mathrm{H}), 8.28(\mathrm{~d}, \mathrm{~J}=8.8 \mathrm{~Hz}, 2 \mathrm{H}) . \operatorname{MS}-\operatorname{ESI}(\mathrm{m} / \mathrm{z}): 415.4[\mathrm{M}+1]^{+}$. Anal. $\left(\mathrm{C}_{20} \mathrm{H}_{18} \mathrm{~N}_{2} \mathrm{O}_{6} \mathrm{~S}\right): \mathrm{C}, \mathrm{H}, \mathrm{N}$.

[3-Amino-5-(3-nitrophenyl)-thiophen-2-yl]-(3,4,5-trimethoxyphenyl)-methanone

$(5 \mathbf{s})$.

Following general procedure B, compound 5s was isolated as a yellow solid. Yield: $40 \%$, mp

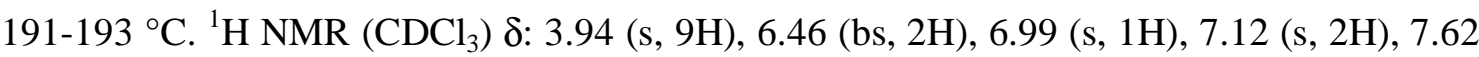


(t, J=7.8 Hz, 1H), $7.88(\mathrm{dd}, \mathrm{J}=7.8$ and $2.2 \mathrm{~Hz}, 1 \mathrm{H}), 8.20(\mathrm{dd}, \mathrm{J}=7.8$ and $2.2 \mathrm{~Hz}, 1 \mathrm{H}), 8.46(\mathrm{~d}$, $\mathrm{J}=2,2 \mathrm{~Hz}, 1 \mathrm{H})$. MS-ESI $(\mathrm{m} / \mathrm{z}): 415.2[\mathrm{M}+1]^{+}$. Anal. $\left(\mathrm{C}_{20} \mathrm{H}_{18} \mathrm{~N}_{2} \mathrm{O}_{6} \mathrm{~S}\right): \mathrm{C}, \mathrm{H}, \mathrm{N}$.

(3-Amino-5-biphenyl-4-yl-thiophen-2-yl)-(3,4,5-trimethoxyphenyl)-methanone

$(5 \mathrm{t})$.

Following general procedure B, compound $\mathbf{5 t}$ was isolated as a yellow powder. Yield: 48\%, mp 79-80 ${ }^{\circ} \mathrm{C} .{ }^{1} \mathrm{H}$ NMR $\left(\mathrm{CDCl}_{3}\right) \delta: 3.93$ (s, 9H), 6.53 (bs, 2H), 6.90 (s, 1H), $7.16(\mathrm{~s}, 2 \mathrm{H})$, $7.46(\mathrm{~m}, 4 \mathrm{H}), 7.66(\mathrm{~m}, 5 \mathrm{H}) . \mathrm{MS}-\mathrm{ESI}(\mathrm{m} / \mathrm{z}): 446.6[\mathrm{M}+1]^{+}$. Anal. $\left(\mathrm{C}_{26} \mathrm{H}_{23} \mathrm{NO}_{4} \mathrm{~S}\right): \mathrm{C}, \mathrm{H}, \mathrm{N}$.

Growth inhibitory activity. Murine leukemia L1210, murine mammary carcinoma FM3A and human T-lymphocyte Molt 4 and CEM cells were suspended at 300,000-500,000 cells/mL of culture medium, and $100 \mu \mathrm{L}$ of a cell suspension was added to $100 \mu \mathrm{L}$ of an appropriate dilution of the test compounds in wells of microtiter plates. After incubation at $37^{\circ} \mathrm{C}$ for two (L1210 and FM3A) or three (Molt 4 and CEM) days, cell number was determined using a Coulter counter. The IC50 was defined as the compound concentration required to inhibit cell proliferation by $50 \%$.

Effects on tubulin polymerization and on colchicine binding to tubulin. Bovine brain tubulin was purified as described previously. ${ }^{1}$ To evaluate the effect of the compounds on tubulin assembly in vitro, ${ }^{2}$ varying concentrations were preincubated with $10 \mu \mathrm{M}$ tubulin in glutamate buffer at $30{ }^{\circ} \mathrm{C}$ and then cooled to $0{ }^{\circ} \mathrm{C}$. After addition of GTP, the mixtures were transferred to $0{ }^{\circ} \mathrm{C}$ cuvettes in a recording spectrophotometer and warmed to $30{ }^{\circ} \mathrm{C}$, and the assembly of tubulin was observed turbidimetrically. The $\mathrm{IC}_{50}$ was defined as the compound concentration that inhibited the extent of assembly by $50 \%$ after a 20 min incubation. The capacity of the test compounds to inhibit colchicine binding to tubulin was measured as described, ${ }^{3}$ except that the reaction mixtures contained $1 \mu \mathrm{M}$ tubulin, $5 \mu \mathrm{M}\left[{ }^{3} \mathrm{H}\right]$ colchicine and $1 \mu \mathrm{M}$ test compound.

Flow cytometric analysis of cell cycle distribution and apoptosis. Cells were washed once in ice-cold PBS and resuspended at $1 \times 10^{6}$ cells per $\mathrm{mL}$ in a hypotonic fluorochrome solution 
containing propidium iodide (Sigma) $50 \mu \mathrm{g} / \mathrm{mL}, 0.1 \%$ sodium citrate and $0.03 \%$ (v/v) nonidet P-40 (Sigma). After a 30 min incubation, the fluorescence of each sample was analyzed as a single-parameter frequency histogram using a FACScan flow cytometer (Becton Dickinson, San Jose, CA). Cell cycle distribution was analyzed with the ModFit LT program (Verity Software House, Inc), and apoptosis was determined by evaluating the percentage of hypodiploid nuclei accumulated in the sub-G0G1 peak.

\section{References}

(1) Hamel, E.; Lin, C.M. Separation of active tubulin and microtubule-associated proteins by ultracentrifugation, and isolation of a component causing the formation of microtubule bundles. Biochemistry 1984, 23, 4173-4184.

(2) Hamel, E. Evaluation of antimitotic agents by quantitative comparisons of their effects on the polymerization of purified tubulin. Cell Biochem. Biophys. 2003, 38, 121 ;

(3) Verdier-Pinard, P.; Lai J.-Y.; Yoo, H.-D.; Yu, J.; Marquez, B.; Nagle D.G.; Nambu, M.; White, J.D.; Falck, J.R.; Gerwick, W.H.; Day, B.W.; Hamel, E. Structure-activity analysis of the interaction of curacin A, the potent colchicine site antimitotic agent, with tubulin and effects of analogs on the growth of MCF-7 breast cancer cells. Mol. Pharmacol. 1998, 53, 62-67. 
Elemental analyses of derivatives 5a-t.

\begin{tabular}{|c|c|c|c|c|c|c|c|c|}
\hline \multirow[t]{2}{*}{ compd } & \multirow[t]{2}{*}{ formula } & \multirow[t]{2}{*}{ MW } & \multicolumn{3}{|c|}{ Calcd } & \multicolumn{3}{|c|}{ Found } \\
\hline & & & $\% \mathrm{C}$ & $\% \mathbf{H}$ & $\% \mathrm{~N}$ & $\% \mathrm{C}$ & $\% \mathbf{H}$ & $\% \mathrm{~N}$ \\
\hline $5 \mathbf{a}$ & $\mathrm{C}_{20} \mathrm{H}_{19} \mathrm{NO}_{4} \mathrm{~S}$ & 369.44 & 65.02 & 5.18 & 3.79 & 64.88 & 5.03 & 3.67 \\
\hline $5 b$ & $\mathrm{C}_{18} \mathrm{H}_{17} \mathrm{NO}_{4} \mathrm{~S}_{2}$ & 375.46 & 57.58 & 4.56 & 3.73 & 57.44 & 4.42 & 3.62 \\
\hline $5 c$ & $\mathrm{C}_{18} \mathrm{H}_{17} \mathrm{NO}_{4} \mathrm{~S}_{2}$ & 375.46 & 57.58 & 4.56 & 3.73 & 57.39 & 4.38 & 3.56 \\
\hline $5 d$ & $\mathrm{C}_{19} \mathrm{H}_{18} \mathrm{~N}_{2} \mathrm{O}_{4} \mathrm{~S}$ & 370.42 & 61.61 & 4.90 & 7.56 & 61.48 & 4.77 & 7.38 \\
\hline $5 e$ & $\mathrm{C}_{20} \mathrm{H}_{18} \mathrm{FNO}_{4} \mathrm{~S}$ & 387.43 & 62.00 & 4.68 & 3.62 & 61.83 & 4.42 & 3.51 \\
\hline $5 f$ & $\mathrm{C}_{20} \mathrm{H}_{18} \mathrm{ClNO}_{4} \mathrm{~S}$ & 403.88 & 59.48 & 4.49 & 3.47 & 59.31 & 4.27 & 3.26 \\
\hline $5 g$ & $\mathrm{C}_{20} \mathrm{H}_{18} \mathrm{ClNO}_{4} \mathrm{~S}$ & 403.88 & 59.48 & 4.49 & 3.47 & 59.29 & 4.28 & 3.31 \\
\hline $5 h$ & $\mathrm{C}_{20} \mathrm{H}_{17} \mathrm{Cl}_{2} \mathrm{NO}_{4} \mathrm{~S}$ & 438.32 & 54.80 & 3.91 & 3.20 & 54.56 & 3.78 & 3.01 \\
\hline $5 i$ & $\mathrm{C}_{20} \mathrm{H}_{18} \mathrm{BrNO}_{4} \mathrm{~S}$ & 448.33 & 53.58 & 4.05 & 3.12 & 53.44 & 3.89 & 2.96 \\
\hline $5 \mathbf{j}$ & $\mathrm{C}_{20} \mathrm{H}_{18} \mathrm{INO}_{4} \mathrm{~S}$ & 495.33 & 48.50 & 3.66 & 2.83 & 48.26 & 3.48 & 2.68 \\
\hline $5 \mathbf{k}$ & $\mathrm{C}_{21} \mathrm{H}_{21} \mathrm{NO}_{4} \mathrm{~S}$ & 383.46 & 65.78 & 5.52 & 3.65 & 65.56 & 5.34 & 3.51 \\
\hline 51 & $\mathrm{C}_{21} \mathrm{H}_{21} \mathrm{NO}_{5} \mathrm{~S}$ & 399.46 & 63.14 & 5.30 & 3.51 & 62.88 & 5.08 & 3.32 \\
\hline $5 \mathrm{~m}$ & $\mathrm{C}_{21} \mathrm{H}_{21} \mathrm{NO}_{5} \mathrm{~S}$ & 399.46 & 63.14 & 5.30 & 3.51 & 62.91 & 5.11 & 3.28 \\
\hline $5 n$ & $\mathrm{C}_{22} \mathrm{H}_{23} \mathrm{NO}_{6} \mathrm{~S}$ & 429.49 & 61.52 & 5.40 & 3.26 & 61.37 & 5.25 & 3.13 \\
\hline 50 & $\mathrm{C}_{23} \mathrm{H}_{25} \mathrm{NO}_{7} \mathrm{~S}$ & 459.51 & 60.12 & 5.48 & 3.05 & 59.88 & 5.24 & 2.95 \\
\hline $5 p$ & $\mathrm{C}_{22} \mathrm{H}_{23} \mathrm{NO}_{5} \mathrm{~S}$ & 413.49 & 63.90 & 5.61 & 3.39 & 63.77 & 5.48 & 3.26 \\
\hline $5 \mathbf{q}$ & $\mathrm{C}_{21} \mathrm{H}_{18} \mathrm{~F}_{3} \mathrm{NO}_{4} \mathrm{~S}$ & 437.43 & 57.66 & 4.15 & 3.20 & 57.43 & 4.02 & 3.03 \\
\hline $5 r$ & $\mathrm{C}_{20} \mathrm{H}_{18} \mathrm{~N}_{2} \mathrm{O}_{6} \mathrm{~S}$ & 437.43 & 57.66 & 4.15 & 3.20 & 57.48 & 4.03 & 3.01 \\
\hline $5 s$ & $\mathrm{C}_{20} \mathrm{H}_{18} \mathrm{~N}_{2} \mathrm{O}_{6} \mathrm{~S}$ & 437.43 & 57.66 & 4.15 & 3.20 & 57.52 & 4.01 & 3.03 \\
\hline
\end{tabular}




\section{Table of Contents Graphic}

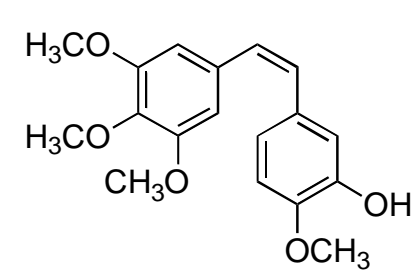

Combretastatin A-4 (CA-4)

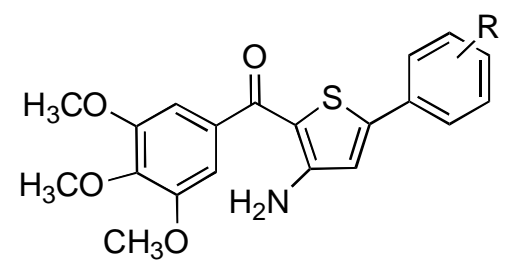

$\mathrm{R}=$ halogen, alkoxy, $\mathrm{Me}, \mathrm{CF}_{3}, \mathrm{NO}_{2}$ 